\title{
The Relationship between Tourette's Syndrome and Infections
}

\author{
Daniela L. Krause* and Norbert Müller
}

Department of Psychiatry and Psychotherapy Ludwig-Maximilians University Munich, Germany

\begin{abstract}
Increasing evidence shows that infections and an activated immune status might be involved in the pathogenesis of tic disorders. Studies discuss the influence of neurotrophic bacteria and viruses on different psychiatric disorders. In addition, signs of inflammation and immunological abnormalities have been described especially in schizophrenia and Tourette's syndrome (tic disorder). Neuroimaging studies revealed increased microglial activation in psychiatric diseases; indicating an inflammatory state of the CNS.

However, it still remains unclear what the underlying mechanism is of how infectious agents could contribute to tic symptoms. One hypothesis is that not only one particular infectious agent causes directly to the disease; instead different (chronic) infections influence the immune balance and are therefore involved in the pathology. In tic disorders, infections with group A streptococci, Borrelia burgdorferi or Mycoplasma pneumoniae seem to be associated with symptoms of the disease. Studies have shown that immunologic treatment improves and prevents the re-occurrence of clinical symptoms in Tourette's syndrome. Also post-infectious events by cross-reactive antibodies(against M-protein) and an altered dopamine rgic(noradrenergic) neurotransmission as well as inflammatory/immunological dysregulations were considered as possible mechanisms to cause symptoms. Another contributing factor to the pathogenesis of these diseases could be an activation of the tryptophan catabolism through infectious agents. Tryptophan functions as a precursor for neurotransmitters like serotonin and becomes degraded to products that can modulate the neurotransmitter balance.
\end{abstract}

A deeper insight into the precise mechanism of how infectious agents influence immune parameter, tryptophan metabolism and the resulting neurotransmitter availability could help finding new therapeutic strategies.

Keywords: Borreliaburgdorferi, immune system, inflammation, Tourette’s syndrome, tryptophan.

\section{BACKGROUND}

Gilles de la Tourette's syndrome (TS), a tic disorder, is characterized by multiple motor and vocal tics, which wax and wane over time [1]. Epidemiological studies have revealed that TS is relatively common (2.9-5.2 cases per $10,000)$ and more prevalent in boys [2]. TS is considered a multi-factorial condition; Recent studies revealed that also environmental factors are involved: Infectious agents like group A $\beta$ haemolytic streptococcal infections seem to be a contributing factor to neuropsychiatric and movement disorders [3]. In addition to the infectious hypothesis for TS, it has been proposed that the involvement of the immune system could be a key component contributing to tic disorders. Beside others, increased levels of pro-inflammatory cytokines [4] and auto-antibodies against basal ganglia have been identified, but are discussed controversially $[5,6]$. Also a neurotransmitter dysbalance involving dopaminergic [7] and serotonergic abnormalities has been reported in TS patients [8]. Another probable mechanism for the influence of infections on the cerebral immune balance could be the activation of the tryptophan catabolism via infectious agents and proinflammatory cytokines. Tryptophan breakdown leads to

*Address correspondence to this author at the Department of Psychiatry and Psychotherapy, Ludwig-Maximilians University, Nussbaumstr. 7, 80336 Munich, Germany; Tel: +49 895160 3397; Fax: +49 895160 4548;

E-Mail: Daniela.Krause@med.uni-muenchen.de neuroactive metabolites that can influence the neurotransmitter balance [9]. Especially in microglia, which are the resident innate immune cells of the brain, tryptophan gets degraded to serotonin and to other products of this metabolism [10], which function either as a NMDA-receptor agonist or antagonist and control the neurotransmitter availability [11]. So far, an imaging study of Behen et al. [12] has identified that in TS there is an abnormal tryptophan metabolism in the cortical and subcortical area. These results are in line with plasma kynurenine levels, that have been shown to be elevated in TS [13]. However, these findings are still controversial in literature. Hoekstra et al. [14] observed an unaltered kynurenine pathway in patients with tic disorders.

Taken this together, TS is a chronic relapsing disorder that can be associated with marked impairment and disability. Although clinical care has improved, a significant number of patients fail to respond adequately or experience intolerable side effects to available treatment options. This review summarizes recent findings related to this topic and integrates available data into a common pathophysiological model. Until now, many questions regarding the association of TS and infections are still unresolved. Discussion continues whether infectious agents and resulting neuroinflammation are an underling cause or a resulting condition in TS.

The purpose of this review is to highlight our new insights into the pathophysiology of TS. We focus on the mechanisms of how infectious agents influence immune 
parameters, tryptophan metabolism and the resulting neurotransmitter availability. And what strategies result from this underling neuroinflammation for future treatment options for TS? The different levels of dysfunction in TS are illustrated in Fig. (1).

\section{INFECTIONS AND TOURETTE'S SYNDROME}

The ethiology of TS is considered to be multi-factorial. In the past years research has focused on infections as a possible contributing factor to the disease or at least to a subgroup of TS patients. It has been observed that children with streptoccoal infections can develop movement disorders such as Sydenham's chorea. These specific movement abnormalities with the clinical phenotype of poststreptococcal tics and obsessive-compulsive disorders are termed pediatric autoimmune neuropsychiatric disorders associated with streptococcal infection (PANDAS) [3].

PANDAS are characterized by the following criteria: 1 . prepubertal onset; 2 . chronic tic disorder or obsessivecompulsive disorder; 3 . sudden periods of severe exacerbations ("saw tooth") - relapsing-remitting course; 4. temporal association of onset and one or more exacerbations with clinical evidence of a streptococcal infections; 5 . the presence of subtle neurological findings such as reduced fine motor coordination or increased motor hyperactivity (but not overt chorea) [15]. However, comparing epidemiological studies and case reports on PANDAS reveals that there is no consistent use of these criteria [16]. Therefore the PANDAS concept itself remains controversial. Like in other poststreptococcal diseases auto-antibodies seem to be involved in the pathology. Also the detection of elevated levels of antibodies to the caudate nucleus and putamen in patients with tic-type hyperkinesias supports the association of the striate area in disease development and provides evidence of the autoimmune nature of the process $[17,18]$. In addition to streptococci, several other infectious agents have been proposed to play an essential role in the pathogenesis of TS. Two case reports revealed that Mycoplasma pneumoniae might be associated with tic symptoms [19, 20]. This assumption was strengthened by a study that compared 29 Tourette patients to healthy controls regarding the Mycoplasma infection rates. The study found significantly more elevated antibody titers against Mycoplasma pneumoniae in TS patients as compared to controls [21]. Furthermore, an association between herpes simplex virus 1 and the exacerbation of tics was stated [22]. Also an acute infection with Borrelia burgdorferi presenting as TS was reported [23]. Our own group also identified infectious agents as contributing factors in TS: A significantly higher rate of TS patients had elevated antibody titers against Chlamydia trachomatis as compared to controls and trend towards a higher prevalence in the Tourette's group was shown for Toxoplasma gondii [24].

Unitl now, none of these infectious agents has been linked consistently with TS. Thus a hypothesis is that infections do not directly cause symptoms of the disease, instead they could contribute to TS by triggering an immune response. Therefore the observations of a dysbalanced immune system will be discussed for TS.

\section{IMMUNE PARAMETERS IN TOURETTE'S SYN- DROME AND POSSIBLE UNDERLYING MECHA- NISMS OF THE ASSOCIATION OF TS AND INFEC- TIOUS AGENTS}

In TS patients nonspecific markers of immune activation have been found to be elevated: The parameter neopterin serves as a biomarker of cell-mediated immunity. It is produced by human monocytes/macrophages during Th1-type immune responses, after stimulation with interferon- $\gamma$. Increased levels of neopterin have been observed in association

\section{Levels of dysfunction in Tourette's syndrome - a psychoneuro-immunological model}

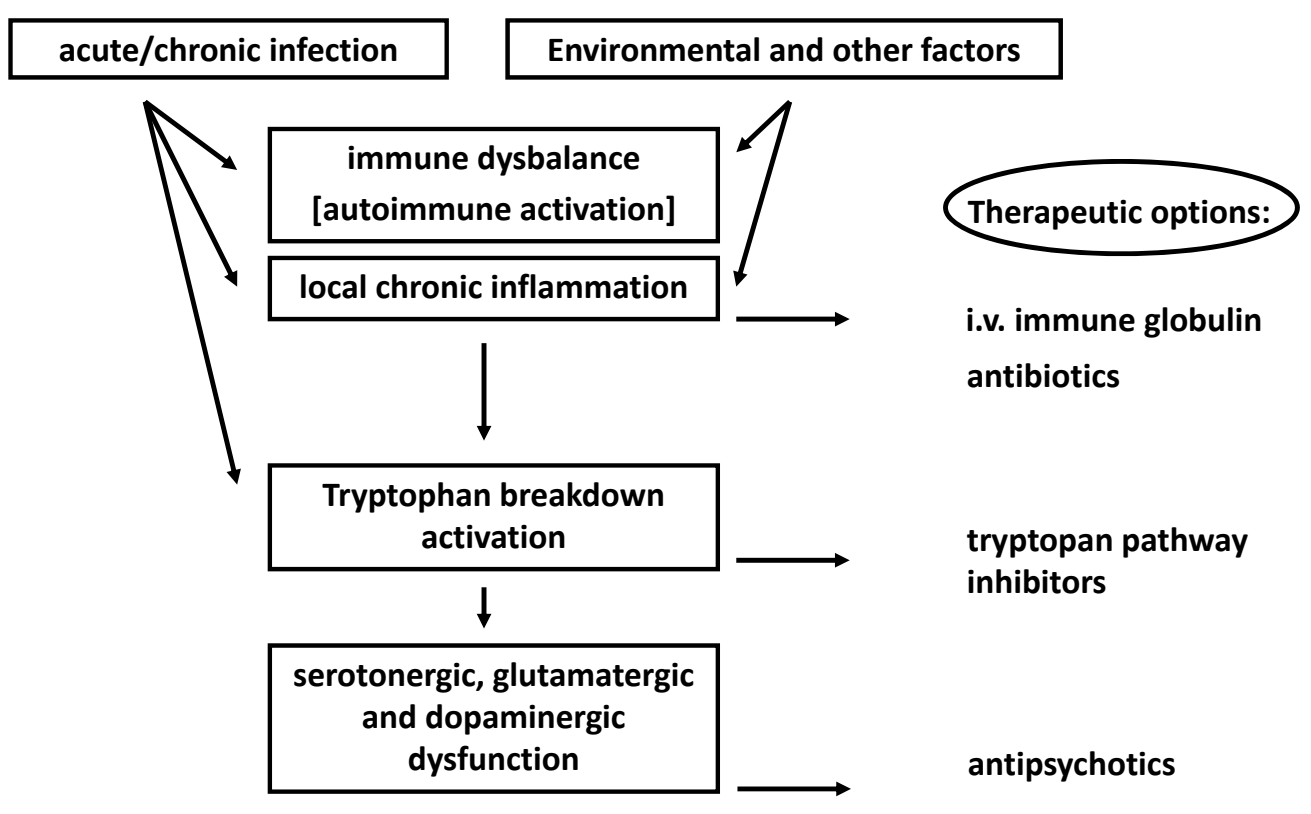

Fig. (1). 
with several autoimmune diseases [25, 26]. Also in TS, neopterin levels at baseline were elevated in patients compared to healthy controls [13]. Another marker is c-reactive protein (CRP), which is an acute-phase protein. It gets secreted mainly in the liver and is modulating inflammatory responses, although the exact mechanism remains controversial. Luo et al. tried to identify a biomarker from patients with serologically defined PANDAS. The authors observed that at baseline, patients demonstrated significantly higher levels of D8/17-reactive cells (a marker ofrheumatic fever) and neopterin, compared with control subjects,but there was no consistent pattern of change when exacerbation time points were compared with baseline or follow-up time points. Unlike neopterin levels, a comparison of CRP levels for patients versus control subjects at baseline indicated no significant difference between TS and controls [27].

Also cytokines have been proposed to be involved in TS. Leckmann et al. found that interleukin (IL)-12 and tumor necrosis factor alpha concentrations at baseline were elevated in patients compared with control subjects. Both of these markers were further increased during periods of symptom exacerbation[4].

Also other immunologic studies of early-onset OCD and tic disorders have found and focused on the link between newly acquired streptococcal infections and symptom exacerbations [15, 27, 28] and the presence of potentially pathogenic antineural antibodies [29]. However, it remains controversial which parallels can be drawn from peripheral cytokines to processes in the central nervous system (CNS). It is known that peripheral cytokines influence brain cells through various mechanisms such as passive transport into the CNS, second messengers and active transport during pathological conditions [30, 31]. In some patients with TS, Bos-Veneman et al. showed that they have decreased serum immunoglobulin (Ig) G3, and possibly also IgM levels, though only few patients had fully expressed Ig immunodeficiency as compared to controls [32].

One explanation of how an altered immune balance caused by infectious agents could contribute to psychiatric symptoms is the 'kindling hypothesis', which is used to convey the conceptof the development of persistent hypersensitivity to a given stimulus. While regularly a certain stimulus is mandatory for the initiation of the immune response, i.e. the release of cytokines and other mediators of immune activation, kindling is associated with an increased release of cytokines after re-exposure to the same stimulus or a weaker stimulus is necessary for the same amount of activation [33].

So, is the connection between infectious agents and immune system relevant for disease pathology? New acquired or chronic neurotrophic infections can influence the immune balance and cause neuroinflammation and/or persistent immune changes. The question is how these immune changes might contribute to clinical symptoms?

Therefore the characteristics of neuroinflammtion need to be taken into consideration:

Inflammation in the brain is characterized by activation of glial cells (mainly microglia and astrocytes) and expression of key inflammatory mediators as well as neurotoxic free radicals. Not much is known so far about the role of neuroinflammation in TS. However, this tic disorder shows a lot of pathophysiological parallels to other psychiatric diseases (e.g. considering the neurotransmitter dysbalance). It has been suggested that neuroinflammation is associated with neurodegenerative disorders - both acute (e.g. stroke, injury) and chronic (e.g. multiple sclerosis, Alzheimer's disease). In this context, microglia cells play a crucial role and therefore microglia and cytokines have been extensively studied in these conditions. In the CNS, microglia are the resident phagocytes of the innate immune system. In an activated state, microglia produce various pro-inflammatory cytokines and other immune mediators that create a neurotoxic milieu leading to disease progression [34, 35]. Microglia are one of three glial cell types in the CNS. They play an important role as immunocompetent and phagocytic cells in the event of injury and disease. Del Rio Hortega determined in 1927 that microglia belong to a distinct glial cell type apart from astrocytes and oligodendrocytes [36]. Since the 1970s there has been wide recognition that microglia are immune effectors in the CNS that respond to pathological conditions and participate in initiation and progression of neurological and psychiatric disorders by releasing potentially cytotoxic molecules such as pro-inflammatory cytokines, reactive oxygen intermediates, proteinases, and complement proteins [37]. This means that their phagocytic function can be beneficial while their inflammation related functions might be detrimental for neuropsychiatric diseases.

A probable mechanism of how infections can influence the cerebral immune balance could be the activation of the tryptophan catabolism via infectious agents and elevated proinflammatory cytokines [9]. In the CNS tryptophan degradation takes place especially in microglia (also partly in astrocytes and neurons). The essential amino acid tryptophan gets degraded either to serotonin or over the kynurenine pathway (KP) to other products [10], which function either as a NMDA- receptor agonist or antagonist and control the neurotransmitter availability [11]. The activation of this KP has been shown to play an important role in the pathophysiology of neuropsychiatric disorders (e.g. schizophrenia) [38]. Also for TS, Behen et al. have identified in an imaging study that TS patients have an abnormal tryptophan metabolism in the cortical and subcortical area [12].

Considering the involvement of neuroinflammation in the course of TS, it strengthens the association between infectious agents activating the tryptophan breakdown either directly or through resulting pro-inflammatory cytokines and therefore influencing the neurotransmitter balance.

\section{NEW THERAPEUTIC OPTIONS FOR TOURETTE'S SYNDROME}

In TS several immunologic changes have been identified. Therefore the question of a possible therapy targeting specific immune parameters has been raised. This idea is further strengthened by the finding of auto-antibodies in TS. Zykov et al. showed that antibodies to caudate nucleus proteins were detected in patients with Tourette's syndrome (the caudate nucleus is a structure, which is functionally important for the appearance of hyperkinesias). The detection of neuroantibodies suggests the possible participation of auto- 
immune mechanisms in the pathogenesis of this disease [39]. The presence of neuroantibodies could be an evidence for impairment of the functions of the blood-brain barrier, which regulates the release of elements of neural structures into the bloodstream, their contact with immune system cells, and the transport of neuroantibodies to the CNS and subsequent influencing the neurotransmitter balance. The precise mechanism of the appearance of autoimmune processes in various nervous system diseases remains a subject of investigation and still it is unclear whether these autoimmune processes are consistently part of TS pathology. So far studies remain controversial: Brilot et al. found serum autoantibodies that bind to neuronal cell surface antigens are present in Sydenham chorea but not in PANDAS or TS. The findings of this group weaken the autoantibody hypothesis in PANDAS and TS [40].

Thus, is the observation of antibodies to caudate nucleus proteins a justification to treat TS patients with immunoglobulins? First studies are showing positive effects after the administration of immunoglobulins to patients with TS in the treatment of tic-type hyperkinesias and provide evidence of the involvement of autoimmune mechanisms inducing damage to the dopaminergic system of the striatum. One case report demonstrated that a 14-year-old boy with TS, familial susceptibility, and an increase in the antibody titer of streptococcal antigens was treated with 75 immunoglobulins i.v. over 5 days. Response to this therapy was good regarding motor tics, vocal tics, and behavioural symptoms such as disturbed impulse control which still persisted after 9 months [41].

Other studies addressed the possibility of using immunoglobulins for intravenous administration and plasma exchange in the treatment of TS. Perlmutter et al. wanted to test if post-streptococcal autoimmunity is the cause of the tic exacerbations. In this case, children might respond to immunomodulatory treatments. Patients received either plasma exchange or i.v. immunoglobulins or i.v. placebo. The authors found that the i.v. immunoglobulin group and the plasma-exchange group showed striking improvements in obsessive-compulsive symptoms, anxiety and overall functioning. Tic symptoms were also significantly improved by plasma exchange compared to placebo. The treatment gains were maintained at one year, with $82 \%$ of children "much" or "very much" improved over baseline [42].

The long-term effects of the i.v. immunoglobulins might be associated with the immunomodulatory actions of normal antibodies on T-lymphocytes and macrophages, as well as Blymphocyte functions and their regulatory actions on the membrane-damaging components of the complement system. Therefore the suggestion that autoimmune mechanisms are involved in the pathogenesis of tic-type hyperkinesias might allow widening of the therapeutic approach to TS in the future.

Taken this together, immunotherapy can be effective in a case in which antibodies to caudate nucleus proteins are detected, which is evidence for the pathogenetic approach of this therapy and further supports the hypothesis that there is an autoimmune variant of tic-type hyperkinesias. However, data on these therapies remains very limited and maybe these new therapeutic options are only applicable to a subset of TS patients.

The discussed infectious and immunologic abnormalities in TS indicate that infectious agents and immune parameters might influence the cerebral neurotransmitter balance via the tryptophan catabolism. The association of infections and the kynurenic pathway could have therapeutic implications, as at present inhibitors of certain metabolites of this pathway are available [9].

\section{CONFLICT OF INTEREST}

The authors confirm that this article content has no conflicts of interest.

\section{ACKNOWLEDGEMENTS}

None declared.

\section{REFERENCES}

[1] Jankovic J. Tourette's syndrome. N Engl J Med 2001; 18; 345(16): 1184-92.

[2] Tanner CM, Goldman SM, Lyons KE, et al. Essential tremor in twins: an assessment of genetic vs environmental determinants of etiology. Neurology 2001; 23; 57(8): 1389-91.

[3] Mell LK, Davis RL, Owens D. Association between streptococcal infection and obsessive-compulsive disorder, Tourette's syndrome, and tic disorder. Pediatrics 2005; 116(1): 56-60.

[4] Leckman JF, Katsovich L, Kawikova I, et al. Increased serum levels of interleukin-12 and tumor necrosis factor-alpha in Tourette's syndrome. Biol Psychiatry 2005; 15; 57(6): 667-73.

[5] Morris CM, Pardo-Villamizar C, Gause CD, Singer HS. Serum autoantibodies measured by immunofluorescence confirm a failure to differentiate PANDAS and Tourette syndrome from controls. J Neurol Sci 2009; 15; 276(1-2): 45-8.

[6] Snider LA, Swedo SE. PANDAS: current status and directions for research. Mol Psychiatry 2004; 9(10): 900-7.

[7] Krause KH, Dresel S, Krause J, Kung HF, Tatsch K, Lochmuller H. Elevated striatal dopamine transporter in a drug naive patient with Tourette syndrome and attention deficit/ hyperactivity disorder: positive effect of methylphenidate. J Neurol 2002; 249(8): 1116-8.

[8] Steeves TD, Fox SH. Neurobiological basis of serotonin-dopamine antagonists in the treatment of Gilles de la Tourette syndrome. Prog Brain Res 2008; 172: 495-513.

[9] Costantino G. New promises for manipulation of kynurenine pathway in cancer and neurological diseases. Expert Opin Ther Targets 2009; 13(2): 247-58.

[10] Heyes MP, Achim CL, Wiley CA, Major EO, Saito K, Markey SP. Human microglia convert l-tryptophan into the neurotoxin quinolinic acid. Biochem J 1996; 1; 320 ( Pt 2): 595-7.

[11] Muller N, Schwarz M. Schizophrenia as an inflammation-mediated dysbalance of glutamatergic neurotransmission. Neurotox Res 2006; 0(2): 131-48.

[12] Behen M, Chugani HT, Juhasz C, et al. Abnormal brain tryptophan metabolism and clinical correlates in Tourette syndrome. Mov Disord 2007; 5; 22(15): 2256-62.

[13] Rickards H, Dursun SM, Farrar G, Betts T, Corbett JA, Handley SL. Increased plasma kynurenine and its relationship to neopterin and tryptophan in Tourette's syndrome. Psychol Med 1996; 26(4): 857-62.

[14] Hoekstra PJ, Anderson GM, Troost PW, Kallenberg CG, Minderaa RB. Plasma kynurenine and related measures in tic disorder patients. Eur Child Adolesc Psychiatry 2007; 16 Suppl 1: 71-7.

[15] Swedo SE, Leonard HL, Garvey M, et al. Pediatric autoimmune neuropsychiatric disorders associated with streptococcal infections: clinical description of the first 50 cases. Am J Psychiatry 1998; 155(2): 264-71.

[16] Murphy TK, Snider LA, Mutch PJ, et al. Relationship of movements and behaviors to Group A Streptococcus infections in elementary school children. Biol Psychiatry 2007; 1; 61(3): 279-84. 
[17] Hallett JJ, Harling-Berg CJ, Knopf PM, Stopa EG, Kiessling LS. Anti-striatal antibodies in Tourette syndrome cause neuronal dysfunction. J Neuroimmunol 2000; 1; 111(1-2): 195-202.

[18] Singer HS, Loiselle CR, Lee O, Minzer K, Swedo S, Grus FH. Anti-basal ganglia antibodies in PANDAS. Mov Disord 2004; 19(4): 406-15

[19] Ercan TE, Ercan G, Severge B, Arpaozu M, Karasu G. Mycoplasma pneumoniae infection and obsessive-compulsive disease: a case report. J Child Neurol 2008; 23(3): 338-40.

[20] Muller N, Riedel M, Forderreuther S, Blendinger C, Abele-Horn M. Tourette's syndrome and mycoplasma pneumoniae infection. Am J Psychiatry 2000; 157(3): 481-2.

[21] Muller N, Riedel M, Blendinger C, Oberle K, Jacobs E, AbeleHorn M. Mycoplasma pneumoniae infection and Tourette's syndrome. Psychiatry Res 2004; 15; 129(2): 119-25.

[22] Budman CL, Kerjakovic M, Bruun RD. Viral infection and tic exacerbation. J Am Acad Child Adolesc Psychiatry 1997; 36(2): 162.

[23] Riedel M, Straube A, Schwarz MJ, Wilske B, Muller N. Lyme disease presenting as Tourette's syndrome. Lancet 1998; 7; 351(9100): 418-9.

[24] Krause D, Matz J, Weidinger E, et al. Association between intracellular infectious agents and Tourette's syndrome. Eur Arch Psychiatry Clin Neurosci 2010; 260(4): 359-63.

[25] Berdowska A, Zwirska-Korczala K. Neopterin measurement in clinical diagnosis. J Clin Pharm Ther 2001; 26(5): 319-29.

[26] Giovannoni G, Lai M, Kidd D, Thorpe JW, et al. Daily urinary neopterin excretion as an immunological marker of disease activity in multiple sclerosis. Brain 1997; 120 (Pt 1): 1-13.

[27] Luo F, Leckman JF, Katsovich L, et al. Prospective longitudinal study of children with tic disorders and/or obsessive-compulsive disorder: relationship of symptom exacerbations to newly acquired streptococcal infections. Pediatrics 2004; 113(6): e578-85.

[28] Murphy TK, Sajid M, Soto O, et al. Detecting pediatric autoimmune neuropsychiatric disorders associated with streptococcus in children with obsessive-compulsive disorder and tics. Biol Psychiatry 2004; 55(1): 61-8.

[29] Kiessling LS, Marcotte AC, Culpepper L. Antineuronal antibodies in movement disorders. Pediatrics 1993; 92(1): 39-43.
[30] Kronfol Z, Remick DG. Cytokines and the brain: implications for clinical psychiatry. Am J Psychiatry 2000; 157(5): 683-94.

[31] Watkins LR, Maier SF, Goehler LE. Cytokine-to-brain communication: a review \& analysis of alternative mechanisms. Life Sci 1995; 57(11): 1011-26.

[32] Bos-Veneman NG, Olieman R, Tobiasova Z, et al. Altered immunoglobulin profiles in children with Tourette syndrome. Brain Behav Immun 2011; 25(3): 532-8.

[33] Frank MG, Baratta MV, Sprunger DB, Watkins LR, Maier SF. Microglia serve as a neuroimmune substrate for stress-induced potentiation of CNS pro-inflammatory cytokine responses. Brain Behav Immun 2007; 21(1): 47-59.

[34] Akiyama H, Barger S, Barnum S, et al. Inflammation and Alzheimer's disease. Neurobiol Aging 2000; 21(3): 383-421.

[35] Wyss-Coray T. Inflammation in Alzheimer disease: driving force, bystander or beneficial response? Nat Med 2006; 12(9): 1005-15.

[36] del Rio-Hortega P. Art and artifice in the science of histology. 1933. Histopathology 1993; 22(6): 515-25.

[37] Block ML, Zecca L, Hong JS. Microglia-mediated neurotoxicity: uncovering the molecular mechanisms. Nat Rev Neurosci 2007; 8(1): 57-69.

[38] Myint AM, Schwarz MJ, Steinbusch HW, Leonard BE. Neuropsychiatric disorders related to interferon and interleukins treatment. Metab Brain Dis 2009; 24(1): 55-68.

[39] Zykov VP, Shcherbina AY, Novikova EB, Shvabrina TV. Neuroimmune aspects of the pathogenesis of Tourette's syndrome and experience in the use of immunoglobulins in children. Neurosci Behav Physiol 2009; 39(7): 635-8.

[40] Brilot F, Merheb V, Ding A, Murphy T, Dale RC. Antibody binding to neuronal surface in Sydenham chorea, but not in PANDAS or Tourette syndrome. Neurology 2011; 26; 76(17): 1508-13.

[41] Muller N, Riedel M, Erfurth A, Moller HJ. [Immunoglobulin therapy in Gilles de la Tourette syndrome]. Nervenarzt 1997; 68(11): 914-6.

[42] Perlmutter SJ, Leitman SF, Garvey MA, et al. Therapeutic plasma exchange and intravenous immunoglobulin for obsessivecompulsive disorder and tic disorders in childhood. Lancet 1999; 2; 354(9185): 1153-8.

This is an open access article licensed under the terms of the Creative Commons Attribution Non-Commercial License (http://creativecommons.org/licenses/by-nc/3.0/) which permits unrestricted, non-commercial use, distribution and reproduction in any medium, provided the work is properly cited. 